\title{
A Brief Analysis of the Strategic Life Curve -- A Reflection on the Theory of Strategic Turning Point
}

\author{
Shiran Li*, Xinghua Xu \\ School of Business \\ Guizhou university of finance and economics \\ Guiyang, China \\ 2691600120@qq.com
}

\begin{abstract}
The speed of interaction between enterprises is getting faster and faster, and the resources, information and other factors are changing with each passing day. It is difficult for enterprise managers to make a correct judgment on the strategic process of the enterprise in this complicated environment. Therefore, we need to re-examine the corporate strategy and shift the focus of the focus to the strategy itself. This paper proposes a strategic life curve based on the study of the strategic turning point theory. Aims to explore the strategic life curve and provide a new focus for companies that need to find the right understanding of internal and external in dynamic environment.
\end{abstract}

Keywords-Strategic management; Strategic life curve; Strategic effectiveness

\section{INTRODUCTION}

From the perspective of the development of strategic management theory, gradually shift from static strategy to dynamic strategy. Although the strategic research is divided into different stages and factions, their common understanding is that the enterprise strategy is a process. The most important thing in this process is the accurate grasp of the internal and external environment of the enterprise, the precise positioning of its own strategy and its Control and evaluation of the strategy implementation process. However, for the strategy itself, it has its own life trajectory. This trajectory is determined on the one hand by the strategic environment during the implementation of the strategy, and on the other hand by the internal development conditions of the enterprise. The description of the strategic trajectory is a good summary of the strategic research, and can also provide new ideas for the future research of strategic management.

\section{Strategic Turning Point Theoty}

Andrew S. Grove puts forward a "strategic turning point". He believes that the strategic turning point is to organize the previous strategy to lose its effectiveness in a changing situation. On the one hand, change may mark the beginning of the company's decline, and on the other hand may indicate the opportunity for redevelopment (Fig. 1). In later speeches and articles, Grove repeatedly elaborated on the thinking of the strategic turning point. This thinking is mainly caused by the change of the situation to the external environment of the enterprise, such as the industrial environment, competitors, suppliers, consumers, etc.[1] Source: R , Burgelman,A.Grove.Strategic Dissonance.California Management Review,1996.

The strategic turning point theory is to grasp the new strategic intentions in a dynamic environment. The strategic turning point is based on the result of competition among all parties in a complex environment, that is, the strategic contradiction and the cognition of strategic contradictions. The strategic turning point theory points out the key choices of the enterprise in the development process. The complexity and change of the environment bring about the uncertainty of the internal and external factors of the enterprise, which leads to the inconsistency of the strategic positioning of the enterprise and the strategic measures taken, thus causing the organization. There is a strategic contradiction, and a "strategic turning point" signal will appear at this time.[1]

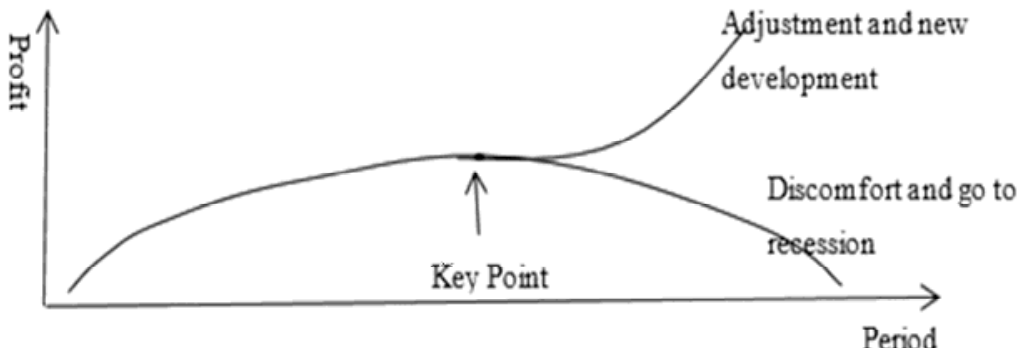

Fig. 1. Strategic turning point. 
In Andrew S. Grove's strategic turning point theory, he focused on one point of focus and how difficult it is for companies to accurately find this major turning point. For the strategy itself, if only focus on its major turning point, it will not be able to effectively control the overall strategy. Strategy is a continuous process. Only by understanding the life track of the strategy itself can we better grasp every important turning point in the strategic process.[2]

\section{STRATEGIC LIFE CURVE}

Based on the above analysis, as with the enterprise life cycle and product life cycle, the corporate strategy should also have a certain life trajectory. Any development strategy adopted by a company cannot remain unchanged for a long time. At the same time, the practical application of each specific strategy It is also impossible to always be in the same stage, but it will change constantly with the passage of time. It should be pointed out that the corporate strategy we are talking about here is the corporate strategy that is in a state of relative market competition. Here, we use strategic effectiveness to measure the changes that a specific strategy has undergone over time. Specifically, it can be divided into six phases, namely: strategic incubation phase, strategic entry phase, strategic growth phase, strategic maturity phase, Strategic change phase and strategic exit phase. For the evaluation of strategy, it is difficult to find a global measurement framework for the strategy. Although there are deep insights in areas such as shareholder value, customer management, human resources, etc., it is more comprehensive with a comprehensive and integrated tool. It is difficult to identify, describe and measure strategies. Robert S. Kaplan and David P. Norton (1992) presented a revolutionary performance measurement system, the Balanced Scorecard, based on their in-depth corporate practice.[4] In the following period, three books have been completed. It can be said that the lifelong research of the two masters provides a more scientific and complete tool for strategic identification, description and measurement for later strategic researchers and practitioners.[2] For the measurement and description of the strategic life curve, we use the results of the two masters to use a balanced scorecard and strategic map to measure the change in the curve of a specific strategy over time (Fig. 2).

Source: Refer to the life cycle curve of tourist destinations.

\section{A. Strategic Gestation Stage}

There are always two considerations when an enterprise chooses a strategy. One is to better cope with the opportunities or challenges brought about by changes in the external environment of the enterprise, and the other is to provide enterprises with better internal tensions to release their own development. In the stage of strategic gestation, managers must first recognize the development situation, the so-called situation, the situation, and the trend. The development situation mentioned here also has two meanings, namely, the external development situation of enterprises such as politics,economy, society, industry, competitors, upstream market and downstream market, and the development stage such as the enterprise, the corporate governance structure, and the enterprise. The inner situation in which culture and business people, finances, and things can match. At this stage, it is important for the management of the company to be fully prepared for the mentality and to make a good plan. It is best to set up a special emergency response team. Warm up the company well and let the company employees understand what to do and recognize their role in a strategy and the status it should achieve and the goals to be achieved.

\section{B. Strategic Entry Stage}

After the strategic plan, the real test is the stage of strategic entry. Generally speaking, due to the reality, the difficulty level of the initial stage of the strategy is unimaginable, and the mental pressure brought to the managers is also the biggest. Once the strategy starts, it is like a car that starts and needs to work together. In the early stage of strategic operation, the instability of the strategic curve may be caused by various reasons. On the one hand, it may be subtle changes in the external market environment of the enterprise, including changes in the social environment, changes in the industry, and reactions from competitors. In many cases, on the other hand, the enterprise itself and the strategic operation are not able to integrate well. For example, the asymmetry and lag of the internal environment of the enterprise such as enterprise structure, culture and resources are inconsistent with the strategic operation. Therefore, at this stage, managers must maintain a high degree of alertness and keen observation, find problems in a timely manner, and solve problems in a timely manner.

\section{Strategic Growth Stage}

Entering the stage of strategic growth, from the performance results, its strategic effectiveness has been stable and rapid. For a correct strategy, its rapid growth is inevitable after it has passed through the period of entry. At this stage, many problems of its strategy and internal and external environment are optimized, the strategy is pioneering, the internal environment of the enterprise adapts to the corporate strategy, and the corporate strategy is better suited to the external environment of the enterprise. The mutual coordination and close cooperation of these three are inevitable. This will enable its strategic effectiveness to be greatly improved. It should be noted that the stage of strategic growth generally does not go through a long period of time, but managers need to have a good grasp of this short spring, whether it is for the control and evaluation of strategy or for future strategic changes. 


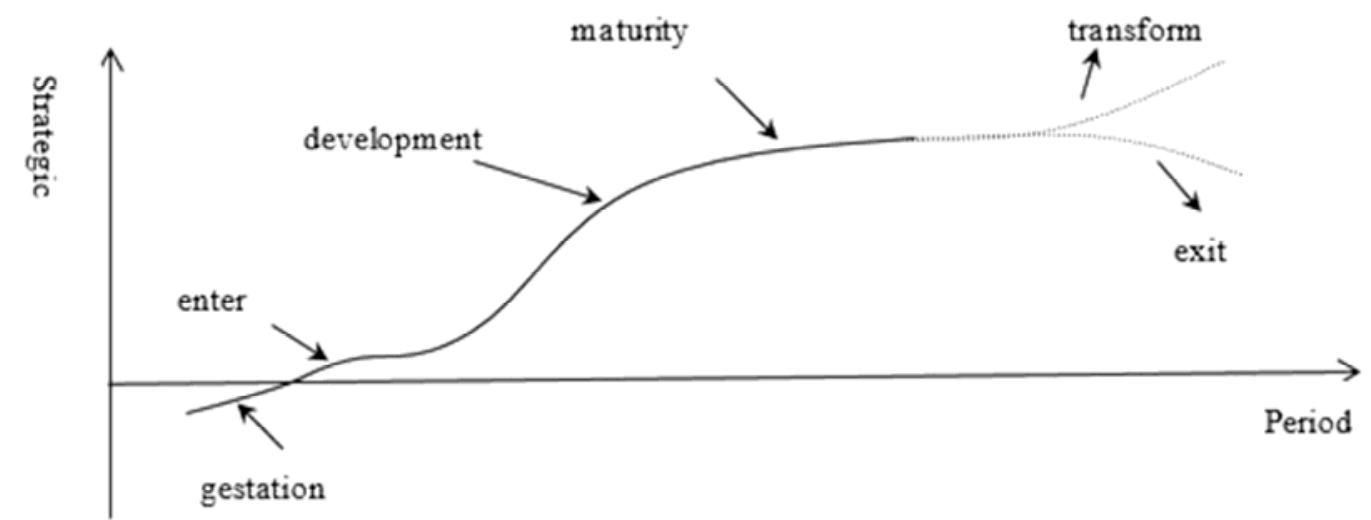

Fig. 2. Strategic life curve.

\section{Strategic Maturity Stage}

A strategy can't be used for life. With the gold development brought by the company's strategic growth, it will first lead to counter-measures from competitors. The way they compete will have a certain impact on the strategic measures taken by the company. The strategic outcome, this shock may not be fatal. Second, it will also lead to strategic replication of followers in the same industry, leading to congestion in the industry. The growth of the final strategy not only brings about the improvement of the efficiency of the enterprise, but whether the enterprise under the strategic growth can fully satisfy the tension under its strategic growth is also the focus that the manager needs to pay attention to. These three main factors bring about a steady and declining trend of its strategic performance curve.

\section{E. Strategic Change Phase}

Strategic change is not simply and completely abandoning the strategy of previous operations. Instead, it is based on the dynamic coordination of the best principles among the environment, strategy, and organization. At the same time, it is necessary to support the cooperation of various elements in the organization to change the enterprise strategy. Initiation, implementation, and sustainable systemic processes. Here, we can refer to the research of relevant scholars. After experiencing the crazy and steady bottleneck of strategic growth, managers should do what they want to control the current reality, or say that good managers should be able to make accurate judgments early and take measures as soon as possible. The strategy and organization are adjusted accordingly to obtain a strategic freshman.

\section{F. Strategic Exit Phase}

If the manager does not recognize the changes in the external environment and the organization, or the inability to act on this change, then the decision to withdraw strategically must be made as early as possible to avoid conflicts between the strategy and the external environment and the organization. Endanger the serious consequences of business life.

The strategic curve is the trajectory of every strategy of the company. This is not to say that all strategies must go through these stages. The strategy described here is only the strategic life under ideal conditions. The strategy may be at any stage, especially in the gestation of strategy and the stage of strategic entry. As an analytical tool, the strategic curve can better solve the problem of abstraction and ambiguity of the strategy, which enables the company management to more intuitively see the stage of the strategy and provide guidance for the company to make decisions.

\section{CONCLUSION AND OUTLOOK}

This paper focuses on grasping the status quo of strategic management development, and proposes the concept of strategic life curve through systematic thinking on strategic turning point theory and classical strategic management theory. The preliminary analysis of the strategic life curve is carried out. Finally, it is pointed out that the strategic life curve is the basic analysis tool for the research and application of strategic management. This paper is only a preliminary discussion of the strategic life curve, and the strategic life curve has extensive research space and research significance in the future.

\section{REFERENCES}

[1] Z. Tang and Y. Zhang, Western Strategic Management Theory, 2rd ed, Beijing, 2008, pp. 35-37.

[2] C.Y. Zhao, "Strategic turning point -- theoretical review and research prospect," Research on science and technology management ,vol. 30(20), pp. 242-245, 2010.

[3] C.Y. Zhao, "Turning point of enterprise strategy: connotation exploration and identification tool design," Research on science and technology management, vol. 2008(02), pp. 4-6, 2008.

[4] R. Kaplan and D. Norton, Balanced Scorecard - action Strategy.1rd ed,Guangzhou, 2013, pp. 88-242.

[5] R. kaplan and D. Norton, Strategic Map - transforming Intangible Assets into Tangible Results, 1rd ed, Guangzhou, 2005, pp. 28-56. 